$11-25-2019$

\title{
Pediatric brainstem gliomas: An institutional experience
}

\author{
Altaf Ali Laghari \\ Aga Khan University, altaf.alilaghari@aku.edu \\ Mirza Zain Baig \\ Rudy L. Ruggles Biomedical Research Institute, USA., baig.zain@aku.edu \\ Muhammad Ehsan Bari \\ Aga Khan University, ehsan.bari@aku.edu \\ Aneela Darbar \\ Aga Khan University, aneela.darbar@aku.edu \\ Naureen Mushtaq \\ Aga Khan University, naureen.mushtaq@aku.edu
}

See next page for additional authors

Follow this and additional works at: https://ecommons.aku.edu/pakistan_fhs_mc_surg_neurosurg

Part of the Neurology Commons, Neurosurgery Commons, Oncology Commons, Pediatrics Commons, and the Surgery Commons

\section{Recommended Citation}

Laghari, A., Baig, M. Z., Bari, M., Darbar, A., Mushtaq, N., Abdullah, U., Khan, D. A. (2019). Pediatric brainstem gliomas: An institutional experience. Asian Journal of Neurosurgery, 1144(1150), 14-4.

Available at: https://ecommons.aku.edu/pakistan_fhs_mc_surg_neurosurg/203 


\section{Authors}

Altaf Ali Laghari, Mirza Zain Baig, Muhammad Ehsan Bari, Aneela Darbar, Naureen Mushtaq, Umme e Hani Abdullah, and Daniya Aziz Khan 


\section{Pediatric Brainstem Gliomas: An Institutional Experience}

\begin{abstract}
Objective: The aim of this study was to analyze the clinical profiles and outcomes of pediatric brainstem gliomas treated at our institute. Methodology: We reviewed the files of 18 pediatric age group patients diagnosed with brainstem glioma at our institution. The following variables were recorded: age, sex, duration of symptoms, date of diagnosis, main clinical symptoms, Karnofsky performance status score, magnetic resonance imaging findings, histopathology findings, details of the treatment given, disease progression, and date of mortality/last follow-up. This data were then transferred to SPSS version 23 which was used for further analysis. Results: The mean age of our cohort was 8.6 years (range 3-15). There were $11(61.1 \%)$ males and $7(38.9 \%)$ females. There were $16(88.9 \%)$ patients with diffuse intrinsic pontine gliomas (DIPGs), $1(5.6 \%)$ patients with exophytic medullary gliomas, and 1 (5.6\%) patient with midbrain/tectal glioma. Mean overall survival (OS) was 9.7 months. Mean progression-free survival (PFS) was 6.3 months. All patients with DIPG eventually passed away from their disease. Patients with DIPG who received radiotherapy had a longer OS and PFS than those who did not (9.8 and 6 months vs. 3.4 and 2.4 months). Diagnostic latency $>1$ month was found to have a statistically significant longer progression-free interval. Conclusion: DIPGs in the pediatric population have a poor prognosis. Radiotherapy serves to increase survival time but is not curative.
\end{abstract}

Keywords: Pediatric brainstem gliomas, radiotherapy, survival

\section{Introduction}

Brainstem glioma is a primary brainstem neoplasm of glial origin. The majority - about $80 \%$ of these tumors comprise a highly malignant tumor of the pons. ${ }^{[1,2]}$ The balance is made up by low-grade gliomas arising elsewhere in the brainstem. ${ }^{[3]}$

Brainstem gliomas have a high incidence in children - accounting for $10 \%-20 \%$ of all childhood primary brain tumors. ${ }^{[1,3-9]}$ It is estimated that between 2007 and 2011, around $350-400$ pediatric cases $(3 / 100,000$ pediatric population) were diagnosed yearly in the USA. ${ }^{[8]}$ In comparison, brainstem gliomas are rarer in the adult population accounting for only $1 \%-2 \%$ of all adult primary brain tumors. ${ }^{[10-13]}$

Tumor behavior and patient clinical course differ according to brainstem localization. ${ }^{[3]}$ Brainstem gliomas are, therefore, classified according to their anatomic location and appearance on magnetic resonance imaging (MRI) T1 and T2 films. ${ }^{[8]}$ In the pediatric age group, they may be stratified into three main categories, namely diffuse

This is an open access journal, and articles are distributed under the terms of the Creative Commons Attribution-NonCommercial-ShareAlike 4.0 License, which allows others to remix, tweak, and build upon the work non-commercially, as long as appropriate credit is given and the new creations are licensed under the identical terms.

For reprints contact: reprints@medknow.com intrinsic pontine glioma (DIPG), exophytic medullary glioma, and midbrain or tectal glioma ${ }^{[3,6,13]}$ This is, in contrast to gliomas of other locations where tumors are categorized based on their histological features according to the World Health Organization (WHO).

The literature on brainstem gliomas is scanty from our part of the globe. To the best of our efforts, we were only able to access four studies that provided some basic rudimentary data on brainstem gliomas from our region. ${ }^{[14-17]}$ Clinical profiles and management were not commented on in these studies. Thus, keeping in mind the scarcity of literature published on our population, we conducted this retrospective review at our institution to analyze the clinical, prognostic, and therapeutic factors involved in the management of brainstem gliomas. To the best of our knowledge, this is the first time such a retrospective analysis and review is coming to light from our region.

\section{Methodology}

We did a retrospective review of all pediatric patients diagnosed with brainstem glioma at the Aga Khan University Hospital

How to cite this article: Laghari $A A$, Baig MZ, Bari E, Darbar A, Mushtaq N, Hani Abdullah UE, et al. Pediatric brainstem gliomas: An institutional experience. Asian J Neurosurg 2019;14:1144-50.

\section{Altaf Ali Laghari, Mirza Zain Baig ${ }^{1}$, Ehsan Bari, Aneela Darbar, Naureen Mushtaq², Umm E Hani Abdullah, Daniyal Aziz Khan ${ }^{3}$}

Departments of Neurosurgery and ${ }^{2}$ Pediatrics, Aga Khan University Hospital, ${ }^{3}$ Medical College, Aga Khan University Hospital, Karachi, Pakistan, ${ }^{1}$ Rudy L. Ruggles Biomedical Research Institute, Danbury, Connecticut, USA
Address for correspondence: Dr. Ehsan Bari,

Department of Neurosurgery, Aga Khan University Hospital, Karachi, Pakistan.

E-mail: ehsan.bari@aku.edu

Access this article online Website: www.asianjns.org

DOI: 10.4103/ajns.AJNS_101_19 Quick Response Code:

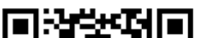


between January 2012 and December 2016 after obtaining approval from the Institution's Ethical Review Committee.

\section{Inclusion criteria}

We designed an inclusion criterion as follows: all pediatric age group patients ( $0-18$ years of age), in whom the center of the tumor bulk, i.e., the epicenter of the tumor resided in the brainstem (medulla oblongata, pons, and midbrain) were included. The diagnosis was based on the histopathological confirmation or a suggestive clinical history supplemented by characteristic MRI findings. Patients who refused to be a part of the study were excluded from our study sample. Furthermore, excluded were all patients with incomplete medical records.

\section{Data collection}

We reviewed files of patients that met our inclusion criteria and collected the following data: patient age, sex, duration of symptoms (described as the time between initiation of symptoms to first contact with a health-care provider), date of diagnosis, main clinical symptoms, Karnofsky performance status (KPS) score, MRI findings (T1- and T2-weighted images in at least two planes before and after gadolinium contrast enhancement), histopathology in cases where a biopsy was done, details of any radiotherapy given, details of any chemotherapy given, details of any surgery done, clinical or radiological disease progression, date of said so progression, and date of mortality/last follow-up.

All data collected were entered and edited manually onto a Microsoft Excel sheet. This was then transferred to the Statistical Package for the Social Sciences Software (SPSS v.23.0, IBM Corp, USA) which was used for further analysis.

\section{Statistical analysis}

The data obtained were analyzed at two levels: descriptive and analytical. Frequencies, percentages, means, and medians were used to describe the characteristics of the study participants.

Overall survival (OS) was the time in months from diagnosis to death or last follow-up. Survival was estimated using the Kaplan-Meier method. Survival differences between variables were compared using the Log-rank test.

Progression-free survival (PFS) was the time in months from diagnosis to disease progression, either clinically or radiologically. It was analyzed the same way as OS.

\section{Results}

\section{Patient demographics and presenting features}

Based on the inclusion criteria, we mentioned previously, the study sample consisted of 18 patients. The mean age of our cohort was 8.6 years (range 3-15), with the majority of patients being male $(61.1 \%)$ [Table 1$]$.
Table 1: Patient demographic and presentation characteristics $(n=18)$

\begin{tabular}{lc}
\hline Characteristic & Number \\
\hline Demographics & \\
Age (years), mean (range) & $8.6(3-15)$ \\
Sex, $n(\%)$ & $11(61.1)$ \\
Male & $7(38.9)$ \\
Female & \\
Presentation & $1(0.1-6)$ \\
Duration of symptoms (month), median (range) & $50(20-80)$ \\
KPS, median (range) & \\
Presenting symptoms, $n(\%)$ & $14(77.8)$ \\
Headache & $14(77.8)$ \\
Ataxia & $13(72.2)$ \\
Diplopia & $8(44.4)$ \\
Dysarthria & $8(44.4)$ \\
Upper limb weakness & $8(44.4)$ \\
Lower limb weakness & $7(38.9)$ \\
Vomiting & $6(33.3)$ \\
Drowsiness & $4(22.2)$ \\
Facial weakness & $4(22.2)$ \\
Dysphagia & $3(16.7)$ \\
Seizures & $2(11.1)$ \\
Decreased vision & $2(11.1)$ \\
Dizziness & $2(11.1)$ \\
Personality change &
\end{tabular}

KPS - Karnofsky performance status

The duration of symptoms had a median value of 1 month (range 3 days -6 months). The most common presenting features were headache, vomiting, seizures, blurry vision, diplopia, facial weakness, dysphagia, dysarthria, ataxia, dizziness, drowsiness, limb weakness, and personality change.

Median Karnofsky performance scores at diagnosis were 50 (range 20-80).

\section{Radiological features}

Based on tumor epicenter, we had 16 (88.9\%) tumors originating from the pons, $1(5.6 \%)$ tumor originating from the cervicomedullary region, and $1(5.6 \%)$ tumor from the midbrain.

Tumors arising from the pons showed diffuse pontine enlargement that appeared hyperintense on T2 weighted images and hypointense on T1-weighted images. Contrast enhancement was seen in 6 patients.

The tumor arising from the cervicomedullary region measured $30 \mathrm{~mm} \times 22 \mathrm{~mm}$. The discrete cystic solid mass caused obliteration of the fourth ventricle leading to hydrocephalus. There was patchy contrast enhancement.

The tumor in the midbrain measured $32.1 \mathrm{~mm} \times 35.4 \mathrm{~mm}$ on sagittal images. It had a cystic component and a contrast-enhancing solid component. It appeared to have a compressive effect on the right internal capsule. 
Subsequent diffusion tensor imaging scan showed abnormal hues identified in the posterior limb of the right internal capsule, right cortical spinal tract, cortical pontine, and cortical bulb tracts.

\section{Classification of tumors}

Based on clinical and radiological profiles, we classified our tumors into three categories. There were $16(88.9 \%)$ cases of DIPG, $1(5.6 \%)$ case of exophytic medullary gliomas, and $1(5.6 \%)$ case of midbrain/tectal glioma.

\section{Surgical procedures}

$6(33.3 \%)$ of patients underwent some surgical intervention. The most commonly performed surgical procedure was ventriculoperitoneal (VP) shunt which was done in $5(22.2 \%)$ patients - all belonging to the DIPG classification of tumors. $2(11.1 \%)$ patients, with tumors belonging to exophytic medullary glioma and midbrain/tectal glioma classifications underwent craniotomy and resection. Our patient with midbrain/tectal glioma developed subdural cerebrospinal fluid (CSF) collection after the surgery. This was initially managed with a subdural drain, but due to the recurrence of collection, he underwent a subdural peritoneal shunt placement.

\section{Pathology}

None of our patients underwent a stereotactic biopsy. Our patients with tumors belonging to the exophytic medullary glioma and midbrain/tectal glioma classifications underwent surgical resection. Histopathological evaluation of both the resected lesions showed the WHO Grade 1 Pilocytic astrocytoma.

\section{Radiotherapy}

11 (61.1\%) patients underwent radiotherapy. Another six patients had also been offered radiation, but due to a scarcity of resources and/or overall poor prognosis of the disease, they chose not to pursue therapy. Our patient with exophytic medullary glioma was not offered radiotherapy due to a favorable surgical profile.

Our patients were offered 30 cycles of radiotherapy to add up to a total dose of $5400 \mathrm{cGY}$.

Four patients out of the 11 who received radiotherapy did not complete their 30 cycles of therapy. This was due to disease progression and death or family decision to discontinue treatment.

\section{Survival}

The mean OS was 9.7 months (range 1-26 months, median 8 months). Mean PFS was 6.3 months (range 1-24 months, median 3 months).

\section{Diffuse intrinsic pontine glioma}

$10(62.5 \%)$ out of 16 patients with DIPG had a clinical disease progression. $6(37.5 \%)$ had both clinical and radiological progression. Mean PFS for patients with DIPG was 4.9 months (range 1-18 months, median 3 months). All patients with DIPG expired from their disease. The mean OS for patients with DIPG was 7.8 months (range 1-18 months, median 8 months).

Patients who received radiotherapy had a longer PFS as opposed to those who did not [Figure 1]. Patients receiving radiation had an average PFS of 6 months, whereas patients who did not receive radiation had an average PFS of only 2.4 months. This difference was found to be statistically significant $(P=0.03)$.

Statistically significant survival benefit was found in patients who received radiotherapy as opposed to those who did not [Figure 2]. The mean OS in DIPG patients who received radiation therapy was 9.8 months compared to 3.4 months in those who did not $(P=0.01)$.

Two patients underwent a VP shunt as well as radiotherapy (PFS: 3 and 9 months, OS: 3.5 and 12 months) and three patients had a VP shunt placement only for symptomatic improvement (PFS: 1, 2, and 3 months and OS: 1, 2, and 8 months).

Female gender, diagnostic latency $>1$ month, KPS score $>60$, and nonenhancing pattern were estimated to be associated with longer OS. However, this was not statistically significant [Table 2].

Diagnostic latency $>1$ month was found to have a statistically significant longer progression free interval [Table 2].

\section{Exophytic medullary glioma}

Our patient with exophytic medullary glioma has not had a disease progression yet and is currently at PFS of 24 months.

\section{Midbrain/tectal glioma}

Our patient with midbrain/tectal glioma had a radiological

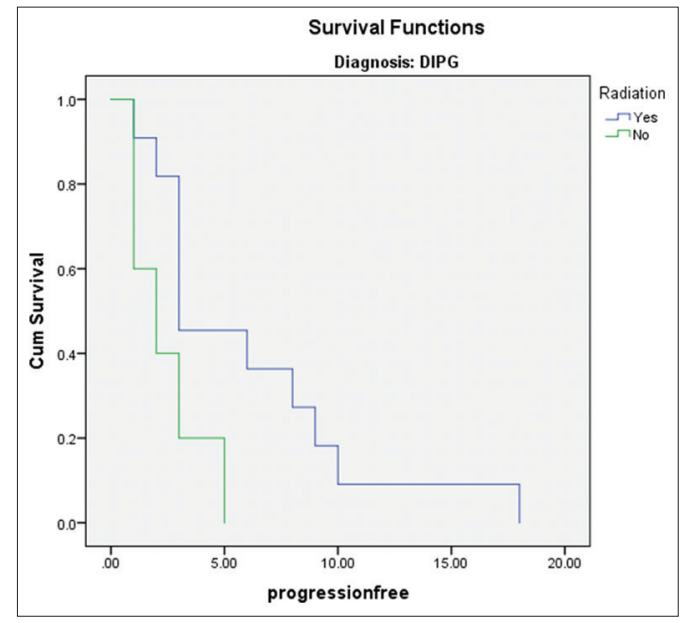

Figure 1: Kaplan-Meier graph depicting progression-free survival in patients with diffuse intrinsic pontine glioma who received radiotherapy versus those who did not 


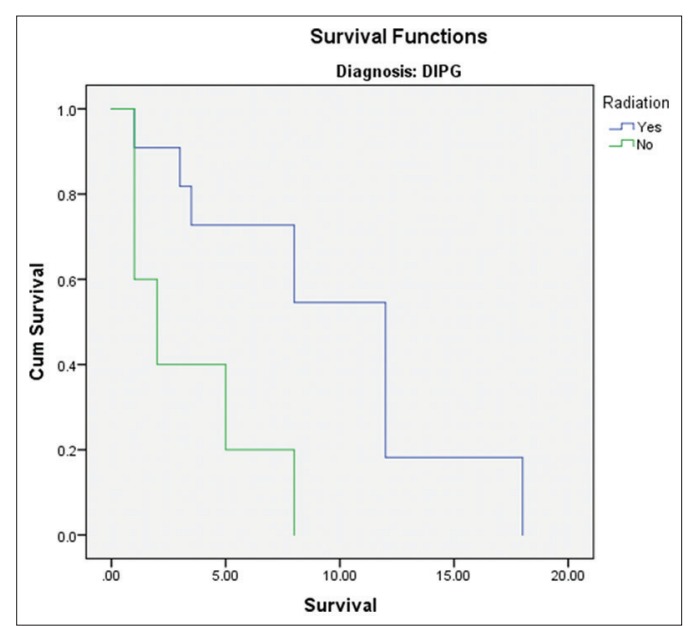

Figure 2: Kaplan-Meier graph depicting overall survival in patients with diffuse intrinsic pontine glioma who received radiotherapy versus those who did not

\begin{tabular}{|c|c|c|c|c|}
\hline & $\begin{array}{c}\text { OS } \\
\text { (months) }\end{array}$ & $\boldsymbol{P}$ & $\begin{array}{c}\text { PFS } \\
\text { (months) }\end{array}$ & $\boldsymbol{P}$ \\
\hline \multicolumn{5}{|l|}{ Gender } \\
\hline Male & 6.6 & 0.36 & 4.3 & 0.43 \\
\hline Female & 9.8 & & 5.8 & \\
\hline \multicolumn{5}{|c|}{ Diagnostic latency (month) } \\
\hline$<1$ & 5.2 & 0.19 & 2.2 & 0.03 \\
\hline$>1$ & 9 & & 6.1 & \\
\hline \multicolumn{5}{|l|}{ KPS } \\
\hline$<60$ & 6.6 & 0.17 & 4.0 & 0.22 \\
\hline$>60$ & 11.4 & & 7.5 & \\
\hline \multicolumn{5}{|l|}{ Contrast } \\
\hline Enhancing & 6.5 & 0.46 & 3.2 & 0.19 \\
\hline Nonenhancing & 8.6 & & 5.9 & \\
\hline \multicolumn{5}{|l|}{ Radiotherapy } \\
\hline Yes & 9.8 & 0.01 & 6.0 & 0.03 \\
\hline No & 3.4 & & 2.4 & \\
\hline
\end{tabular}

OS - Overall survival; PFS - Progression-free survival ; KPS Karnofsky performance status

and clinical progression at 12 and 18 months. She is currently alive at 26 months after the diagnosis.

Table 3 depicts a summary of our patient's clinical course and outcomes.

\section{Discussion}

DIPG formed the majority of tumors in our cohort. These tumors, due to their aggressive clinical course, residence in an eloquent part of the brain and overall poor prognosis as evident from previous literature, as well as our cohort, represent a challenge to pediatric neurosurgeons and oncologists.

DIPGs are the most frequently encountered brainstem gliomas. ${ }^{[2]}$ Although they can arise in any age group, they have a peak onset between 6 and 9 years ${ }^{[3,18]}$ and have a median OS of only 9 months. ${ }^{[9,19]}$ There is an equal predilection for both sexes. ${ }^{[20]}$ It is estimated that $80 \%$ of all gliomas in children are DIPG. ${ }^{[20]}$

Diffuse brainstem gliomas have the classic presentation of the neurological triad: cranial neuropathy (cranial nerve deficits), long tract signs and ataxia. ${ }^{[4,18,20]}$ A very sensitive finding of DIPG is abducens palsy in a young child. ${ }^{[18]}$ This occurred in a number of our patients presenting as strabismus and diplopia.

DIPG is recognized on MRI by diffuse enlargement of the brainstem with or without heterogeneous contrast enhancement. ${ }^{[6,13]}$ They appear hyperintense on T2-weighted images and hypointense on $\mathrm{T}^{\left[{ }^{[3]}\right.}$ These features are so characteristic of this pathology that a histopathological evaluation is not needed for establishing the diagnosis. ${ }^{[3,6,13]}$ The scans of all our DIPG patients demonstrated these findings.

None of our patients underwent a stereotactic biopsy as we felt confident in our diagnosis based on clinical features and characteristic radiology. In the precomputer tomography and MRI eras, stereotactic biopsies used to be a routine procedure for the histological diagnosis of DIPG. This practice was abandoned in the early 1990s due to the heterogeneity of these tumors, the associated morbidity of these biopsy procedures, the prevalence of poor candidates at presentation, limited therapeutic options based on these biopsy results and following the advent of the MRI, the widespread availability of characteristic imaging findings. ${ }^{[20]}$

However, it is now agreed that biopsies of these lesions are relatively safe due to modern neurosurgical techniques and should be considered in the context of clinical trials. Currently, the development of targeted therapies has been hampered by the lack of understanding regarding tumor biology and trials have been conducted based on the misconception that DIPG biology is similar to their adult counterparts and other pediatric supratentorial malignant gliomas. ${ }^{[9]}$ The availability of biopsy material will allow for molecular biology analysis, including whole-genome sequencing, thus potentially allowing for the development of future therapies. ${ }^{[9]}$

The current standard of care for DIPG is fractionated external beam radiotherapy. Radiotherapy serves only to prolong life and does not have a curative purpose as the disease is rapidly fatal. ${ }^{[9,18]}$ Our cohort demonstrates a statistically significant survival benefit among patients who opted for radiotherapy versus those who did not. Radiation may be delivered through a conventional radiotherapy schedule (6 weeks course with once a day treatment with $180 \mathrm{cGY}$ per fraction to deliver a total dose of $5400 \mathrm{cGY}$ as was done with our patients), hyperfractionated radiotherapy schedule (6 weeks course with twice a day treatment with $117 \mathrm{cGY}$ per fraction to deliver a total dose of $7020 \mathrm{cGY}$ ) or hypofractionated radiotherapy schedule (2.6 weeks 


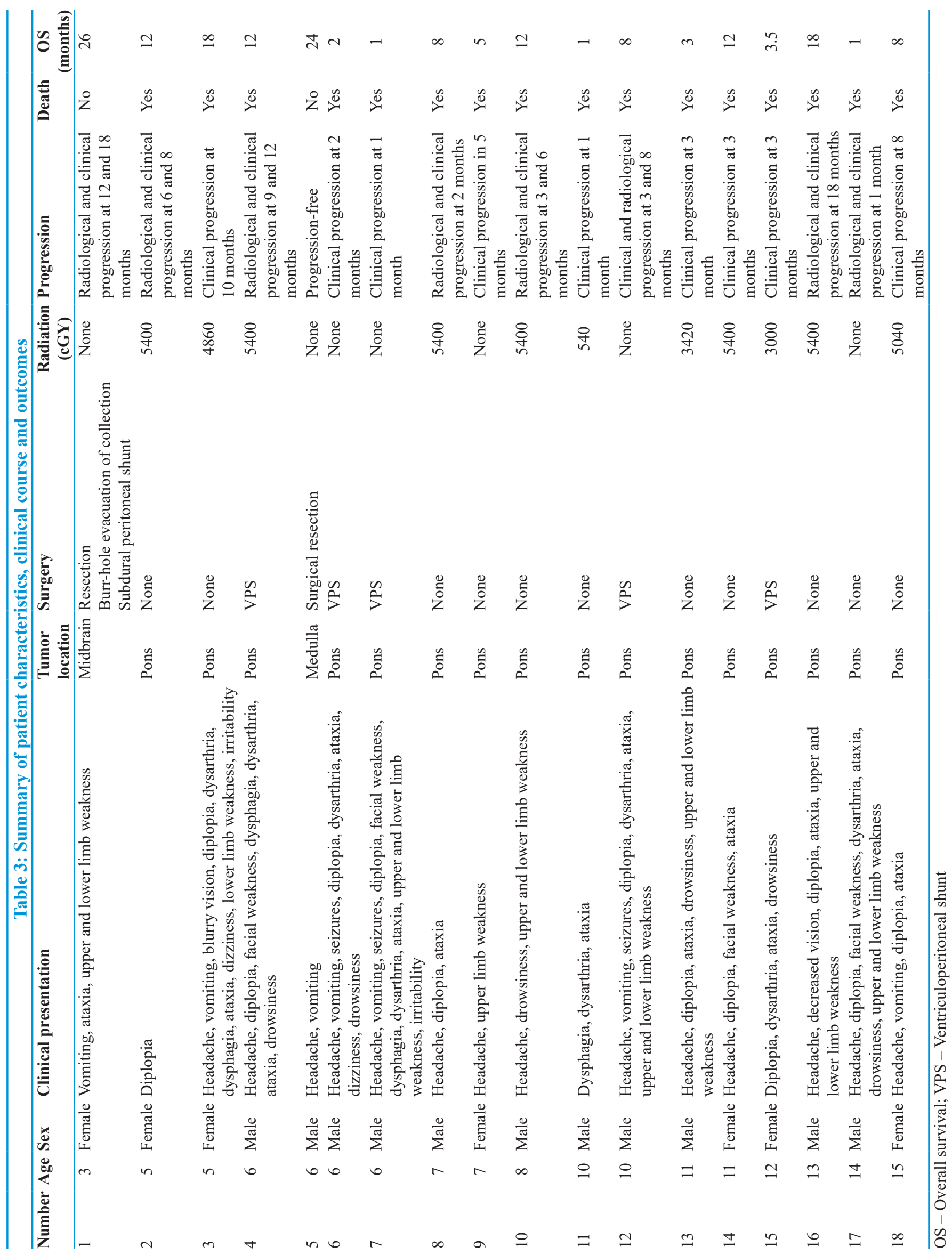


course with $300 \mathrm{cGY}$ per fraction to deliver a total dose of $3900 \mathrm{cGY}$ in 13 fractions). ${ }^{[3,4]}$ A Cochrane review published in 2016, comparing different treatment modalities found no clear evidence regarding differences in OS and PFS between conventional radiotherapy and hypo/hyper fractionated radiotherapy. ${ }^{[4]}$ The shorter hypofractionated radiotherapy schedule, however, has been found to reduce treatment burden while at the same time proving equally efficacious. ${ }^{[18]}$

Currently, there are no proven chemotherapeutic or targeted therapy agents that have been shown to improve PFS and OS. ${ }^{[18]}$ Radiosensitizers such as carbogen and motexafin gadolinium have all found to be ineffective. ${ }^{[18]}$ None of our patients received any chemotherapeutic agent.

DIPG due to their diffuse growth pattern and involvement of important centers of the brainstem are not amenable to surgery. However, five of our DIPG patients had surgical intervention in the form of VP shunt placement. This was due to the development of obstructive hydrocephalus requiring the need for urgent decompression.

On the other hand, focal brainstem gliomas have a subtler and more indolent course often presenting with nonspecific headache and hydrocephalus. ${ }^{[4,7]}$ We had two such patients in our cohort.

Our patient with exophytic medullary glioma presented with a KPS score of 80 and a duration of symptoms of 3.5 months indicating a comparatively slower progression of the disease. Exophytic medullary gliomas are low-grade gliomas with a relatively benign clinical course. ${ }^{[3,6]}$ They arise from the cervicomedullary junction or from the floor of the fourth ventricle and often have a contrast enhancing exophytic portion that may be amenable to surgical resection. ${ }^{[3,6,13]}$ This was done in our case. There may be a need for shunt placement in some patients. ${ }^{[3]}$ Prognostically, these patients have a long-term survival and median OS $>5$ years. ${ }^{[6,13]}$

The third subgroup, midbrain/tectal gliomas also have a relatively benign course. Mean age at diagnosis is 7-10 years. Given their proximity to the cerebral aqueduct, they may often be complicated by hydrocephalus. ${ }^{[6,13]}$ On MRI, they appear as a midbrain lesion that is hypointense on $\mathrm{T} 1$ and hyperintense on T2. They may have marked contrast enhancement. ${ }^{[21]}$ Neurosurgical resection is the treatment of choice for focal midbrain gliomas with the exception of those implicating the tectum. ${ }^{[7]}$ They can usually be managed with CSF diversion with shunt placement or endoscopic third ventriculostomy and periodic radiologic surveillance. ${ }^{[2]}$ Median PFS and OS is beyond the follow-up duration of most studies indicating a good prognosis. $^{[3]}$

Lundar et al. published an institutional series of 15 patients with low-grade midbrain gliomas that were treated with surgical resection. ${ }^{[21]}$ Three patients in their series died, one of whom had a prolonged survival period of 21 years. One patient passed away from acute mesencephalic bleeding 8 months after the initial surgery. And one patient died intraoperatively. Among the 12 surviving patients, stable long-term survival appeared obtainable in at least 9. No patient had rapid tumor progression. Based on these findings, the authors concluded surgical resection as a viable option for focal midbrain gliomas.

Our patient had a KPS at presentation of 80 and a duration of symptoms of 4 months, again highlighting the slow course of the disease. She underwent a craniotomy and resection of her space occupying lesion. Her postoperative scans showed an interval reduction of midbrain lesion. She was readmitted after 2 weeks as she had developed a subdural collection. This was managed with subdural drain placement and evacuation of collection. Recurrence of this collection after another 10 days led to readmission and subsequent management with a subdural peritoneal drain placement. She is currently alive at 26 months after the initial diagnosis.

Whenever a low-grade brainstem glioma cannot be totally resected but there is modest residual disease with minimal neurologic deficits, expectant management with close radiologic surveillance is sufficient. ${ }^{[2]}$ However, in cases of significant and symptomatic residual disease, adjuvant therapy may be offered depending on the efficiency of the said therapy of rescue at tumor relapse and provided the risk of irreversible injury from additional disease progression is greater than the potential toxicity of the adjuvant therapies. ${ }^{[2]}$ Our patient with the focal midbrain glioma had been recommended to receive adjuvant radiotherapy. However, the patient declined the offer. This may have been a reason for the tumor progression later encountered.

\section{Limitations}

Our study looks at the clinical profiles and outcomes of pediatric patients presenting with brainstem glioma that received or did not receive any medical intervention at our center. However, the small cohort of patients due to the rarity of this condition limits the significance of our results. It is, therefore, important to be cautious when interpreting the findings of our paper. A multi-institutional study with a large number of patients would be able to better elucidate the normal progression and outcome of this rare disease.

\section{Conclusion}

DIPGs in the pediatric population have a poor prognosis. Radiotherapy serves to increase survival time but is not curative. In comparison focal low-grade gliomas in the medulla and midbrain are amenable to surgical intervention and generally have a good prognosis with long OS.

Financial support and sponsorship

Nil. 


\section{Conflicts of interest}

There are no conflicts of interest.

\section{References}

1. Green AL, Kieran MW. Pediatric brainstem gliomas: New understanding leads to potential new treatments for two very different tumors. Curr Oncol Rep 2015;17:436.

2. Upadhyaya SA, Koschmann C, Muraszko K, Venneti S, Garton HJ, Hamstra DA, et al. Brainstem low-grade gliomas in children-excellent outcomes with multimodality therapy. J Child Neurol 2017;32:194-203.

3. Grimm SA, Chamberlain MC. Brainstem glioma: A review. Curr Neurol Neurosci Rep 2013;13:346.

4. Hu X, Fang Y, Hui X, Jv Y, You C. Radiotherapy for diffuse brainstem glioma in children and young adults. Cochrane Database Syst Rev 2016;6:CD010439.

5. Oka H, Utsuki S, Tanizaki Y, Hagiwara H, Miyajima Y, Sato K, et al. Clinicopathological features of human brainstem gliomas. Brain Tumor Pathol 2013;30:1-7.

6. Ramos A, Hilario A, Lagares A, Salvador E, Perez-Nuñez A, Sepulveda J. Brainstem gliomas. Semin Ultrasound CT MR 2013;34:104-12.

7. Sun T, Wan W, Wu Z, Zhang J, Zhang L. Clinical outcomes and natural history of pediatric brainstem tumors: With 33 cases follow-ups. Neurosurg Rev 2013;36:311-9.

8. Hassan H, Pinches A, Picton SV, Phillips RS. Survival rates and prognostic predictors of high grade brain stem gliomas in childhood: A systematic review and meta-analysis. J Neurooncol 2017;135:13-20.

9. Puget S, Beccaria K, Blauwblomme T, Roujeau T, James S, Grill J, et al. Biopsy in a series of 130 pediatric diffuse intrinsic pontine gliomas. Childs Nerv Syst 2015;31:1773-80.

10. Hundsberger T, Tonder M, Hottinger A, Brügge D, Roelcke U, Putora PM, et al. Clinical management and outcome of histologically verified adult brainstem gliomas in Switzerland: A retrospective analysis of 21 patients. J Neurooncol 2014;118:321-8.
11. Reithmeier T, Kuzeawu A, Hentschel B, Loeffler M, Trippel M, Nikkhah G. Retrospective analysis of 104 histologically proven adult brainstem gliomas: Clinical symptoms, therapeutic approaches and prognostic factors. BMC Cancer 2014;14:115.

12. Babu R, Kranz PG, Karikari IO, Friedman AH, Adamson C. Clinical characteristics and treatment of malignant brainstem gliomas in elderly patients. J Clin Neurosci 2013;20:1382-6.

13. Lachi PK, Irrakula M, Ahmed SF, Joseph D, Pamidighantam S, Jagannath Rao Naidu KV. Clinical profile and outcomes in brainstem glioma: An institutional experience. Asian J Neurosurg 2015;10:298-302.

14. Irfan A, Qureshi A. Intracranial space occupying lesions - Review of 386 cases. J Pak Med Assoc 1995;45:319-20.

15. Hanif G, Shafqat S. Morphological pattern and frequency of intracranial tumours in children. J Coll Physicians Surg Pak 2004; 14:150-2.

16. Ali M, Ullah W, Jamal B, Alam I, Hussain N. Frequency and pattern of primary pediatric brain tumours: A retrospective study in neurosurgery unit, lady reading hospital Peshawar, Pakistan. KMUJ 2016;8:131-3.

17. Shah SH, Soomro IN, Hussainy AS, Hassan SH. Clinico-morphological pattern of intracranial tumors in children. J Pak Med Assoc 1999;49:63-5.

18. Mathew RK, Rutka JT. Diffuse intrinsic pontine glioma: Clinical features, molecular genetics, and novel targeted therapeutics. J Korean Neurosurg Soc 2018;61:343-51.

19. Vallero SG, Bertin D, Basso ME, Pittana LS, Mussano A, Fagioli F. Diffuse intrinsic pontine glioma in children and adolescents: A single-center experience. Childs Nerv Syst 2014;30:1061-6.

20. Vanan MI, Eisenstat DD. DIPG in children - What can we learn from the past? Front Oncol 2015;5:237.

21. Lundar T, Due-Tønnessen BJ, Egge A, Scheie D, Brandal P, Stensvold E, et al. Neurosurgical treatment of pediatric low-grade midbrain tumors: A single consecutive institutional series of 15 patients. J Neurosurg Pediatr 2014;14:598-603. 